\title{
A chemical shock tube driven by detonation
}

\author{
J. Li • H. Chen - H. Yu
}

Received: 13 February 2011 / Revised: 21 November 2011 / Accepted: 29 February 2012 / Published online: 11 May 2012

(c) Springer-Verlag 2012

\begin{abstract}
A chemical shock tube driven by a detonation driver is described in the present paper. This shock tube can produce a single controlled high-temperature pulse for studies of gas-phase reaction kinetics, but the difficulty associated with the timing for the rupture of diaphragms in the conventional chemical shock tube is overcome, because the detonation wave in the driver section can be predicted correctly and shows a good repeatability. In addition, this shock tube is capable of providing higher temperature conditions for the test gas than the conventional high-pressure shock tube, owing to the inherently high-driving capability of the detonation driver. The feasibility of this shock tube is examined by numerical simulations and preliminary experiments.
\end{abstract}

Keywords Shock tube - Detonation driver . Chemical kinetics study

\section{Introduction}

Experimental studies of reaction kinetics at high temperatures usually require that the test gas is heated rapidly to a desired temperature and pressure, to remain at this reaction condition for few milliseconds, and then to be cooled down so quickly that the reaction in the test gas is frozen, i.e., the studies require that the test gas is processed by a single controlled high-temperate pulse. In a conventional shock tube, the test gas can be heated rapidly by the incident or reflected shock and cooled by the expansion wave generated from the

Communicated by H. Olivier.

J. Li (凶) $\cdot$ H. Chen $\cdot$ H. Yu

Laboratory of High Temperature Gas Dynamics,

Chinese Academy of Sciences, Beijing 100190, China

e-mail: jinping.li@tom.com tube end or shock-interface interaction. The reaction temperature and testing time can be controlled by the operating parameters. The conventional shock tube thus should meet the essential demand of experimental kinetics study of reactions at high temperatures. Early in the 1950s, a number of investigations of chemical kinetics were carried out by use of conventional shock tubes $[1,2]$.

However, in a conventional shock tube (Fig. 1a), there are repeated reflections of the shock wave that subject the test gas to a series of temperature pulses (Fig. 2a). Although the amplitude of the temperature pulses decays gradually, the complex temperature history causes difficulties in subsequent chemical analysis and decreases the reliability of the obtained experimental data, so that the value of conventional shock tubes for chemical kinetics investigations is limited. In order to solve this problem, Glick et al. [3] developed a chemical shock tube (Fig. 1b) by adding a large, evacuated tank separated by an auxiliary diaphragm from the driver section. This tank serves to absorb the reflected shock wave from the end of the driven section, so as to prevent the reactant gas from being reheated (Fig. 2b). The expansion wave that is generated by the rupture of the auxiliary diaphragm is responsible for cooling the test gas. The testing time at high temperature and the cooling rate of the test gas are dependent on the time interval between the rupture of the two diaphragms. It is only when the rupture of the auxiliary diaphragm and the arrival of the head of expansion wave that was generated by the rupture of the main diaphragm take place simultaneously that the maximum testing time and cooling rate are achieved at the same time [3]. This shock tube is impeccable in principle, but it is not easy to precisely control the time interval between the rupture of the two diaphragms in practice.

Another shock tube suitable for chemical kinetics studies is proposed by Lifshitz et al. [4], as shown in Fig. 1c. This shock tube is characterized by a dump tank attached 


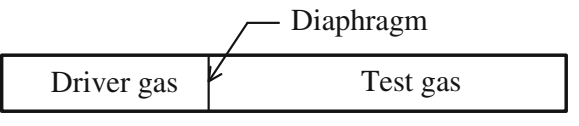

(a) Conventional shock tube

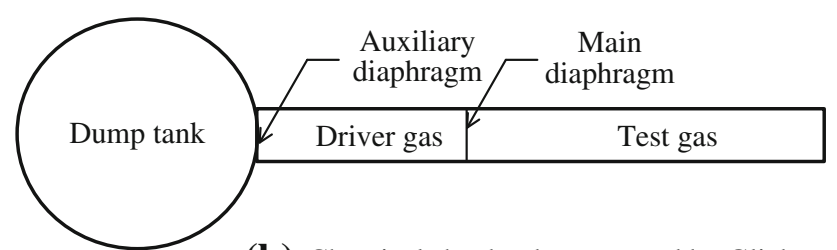

(b) Chemical shock tube proposed by Glick

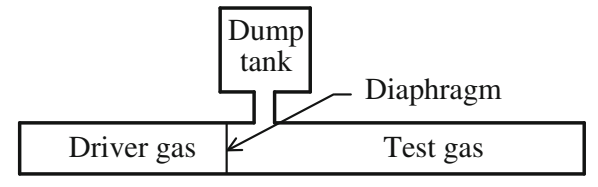

(c) Chemical shock tube proposed by Lifshitz

Fig. 1 Schematic diagrams of conventional and chemical shock tubes

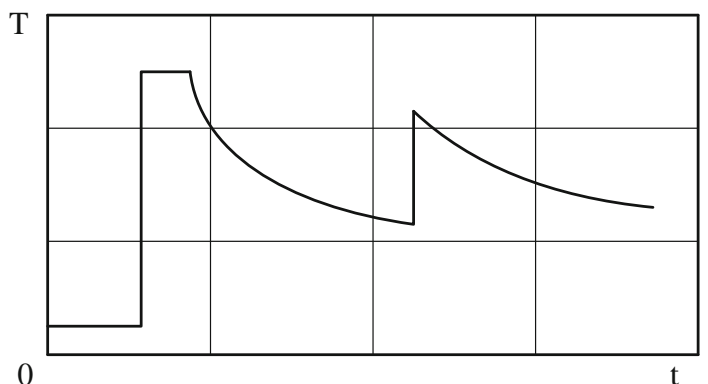

(a) Conventional shock tube

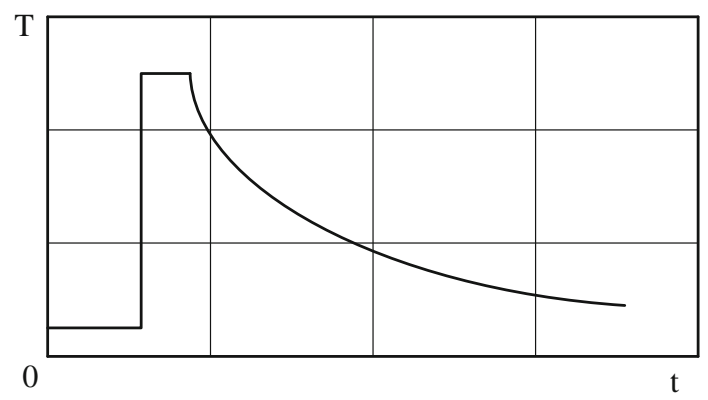

(b) Chemical shock tube

Fig. 2 Temperature histories of the test gas in conventional and chemical shock tube

to the driven section close to the diaphragm, which serves to weaken the strength of a reflected shock wave when it sweeps past the opening to the dump tank and back again. The expansion wave that cools the reactant gas is generated by the interaction between the reflected shock wave and gas interface, which may produce higher cooling rates than the reflected expansion wave from the driver section. Compared with the chemical shock tube proposed by Glick, the one proposed by Lifshitz has a simpler structure, better reproducibility and more convenient operation because of having only one diaphragm, so it is now widely used. However, it cannot eliminate completely the reheating pulse [5], and the behavior of the incident shock wave is more or less influenced by the dump tank.

In the present article, a chemical shock tube driven by a detonation wave is proposed, aiming to overcome the problem associated with the rupture of two diaphragms that was encountered in the shock tube proposed by Glick. In addition, it is expected that higher temperature conditions for the test gas can be obtained with this shock tube to meet the demand of some chemical kinetic studies, such as ionization-recombination kinetics experiments. Several aspects of this shock tube are considered here. First, the concept of this shock tube is introduced. Then, the operating conditions and some limitations of this shock tube are discussed. Finally, the feasibility of this shock tube is examined by numerical simulations and preliminary experiments.

\section{Detonation driver}

The detonation driver, first proposed by Bird [6], has been studied by several investigators [7-9]. Nowadays, it is widely used for shock tubes and tunnels producing high enthalpy flows for aerodynamic testing [10].

Assuming that a closed straight tube is filled with a detonable gas and that a detonation wave is initiated at a closed end which propagates toward the other end, the flow state behind the detonation wave, in terms of the Chapman-Jouguet detonation theory (C-J theory) and the Taylor similarity law [11], can be depicted qualitatively as shown in Fig. 3. The unburnt gas is compressed by the leading front of the detonation wave and releases energy, resulting in the gas pressure, temperature and speed rising abruptly to the C-J values. Simultaneously, a Taylor expansion develops behind the detonation wave in order to match the closed-end boundary condition, by which the burnt gas is gradually decelerated to a stationary state with constant and uniform properties. If a driven section filled with a test gas is connected to the right of the detonation tube, a detonation-driven shock tube is obtained, in which the stationary gas at high temperature and high pressure is the effective driver gas.

Because the gas flow behind the detonation wave is a simple wave flow, the effective pressure of driver gas $P_{\mathrm{e}}$ and sound speed of driver gas $a_{\mathrm{e}}$ for the detonation-driven shock tube are related to the $\mathrm{C}-\mathrm{J}$ values by (see Appendix A)

$a_{\mathrm{e}}=\frac{\left|V_{\mathrm{D}}\right|}{2}$ 


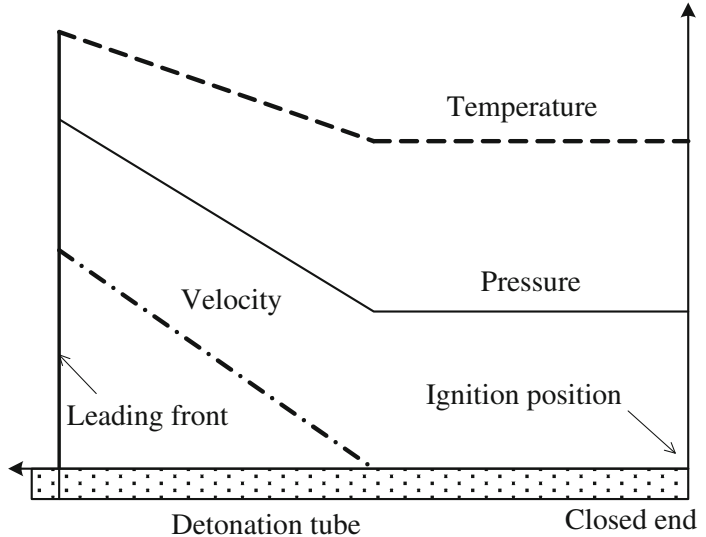

Fig. 3 Schematic diagram of the distribution of detonation wave properties along the detonation tube

$P_{\mathrm{e}}=P_{\mathrm{CJ}}\left(\frac{a_{\mathrm{e}}}{a_{\mathrm{CJ}}}\right)^{\frac{2 \gamma_{\mathrm{D}}}{\gamma_{\mathrm{D}}-1}}$

where subscript CJ denotes the $\mathrm{C}-\mathrm{J}$ state, $V_{\mathrm{D}}$ is the speed of the detonation wave and $\gamma_{\mathrm{D}}$ is the ratio of specific heats of the gas behind the detonation wave.

\section{Detonation-driven chemical shock tube}

\subsection{Wave process in detonation-driven chemical shock tube}

When a detonation driver is introduced into the chemical shock tube, the arrangement is similar to the one proposed by Glick, as shown in Fig. 4, but the wave processes are a little different. The wave process in a detonation chemical shock tube can be described as follows. Direct initiation at the main diaphragm leads to a stable detonation wave in the driver section, behind which the products are at high temperature and pressure. Shortly after initiation, the main diaphragm ruptures and the detonation products exhaust into the driven section, driving the incident shock wave which compresses and heats the test gas. Because the effective driver gas is always the stationary gas, which is defined by the characteristic labeled $e$ in Fig. 4, with constant thermodynamic properties, the incident shock wave acts in the same way as in a conventional shock tube. On arriving at the auxiliary diaphragm, the detonation wave ruptures it and an expansion wave is generated, which serves to weaken the reflected shock wave and can be used to cool the test gas. After entering into the dump section, the detonation wave and the reflected shock wave are mostly trapped within it. Therefore, the test gas can be heated by a single pulse.

From the wave process described above, it can be found that the detonation-driven chemical shock tube conforms to the same principle of preventing the gas from being reheated

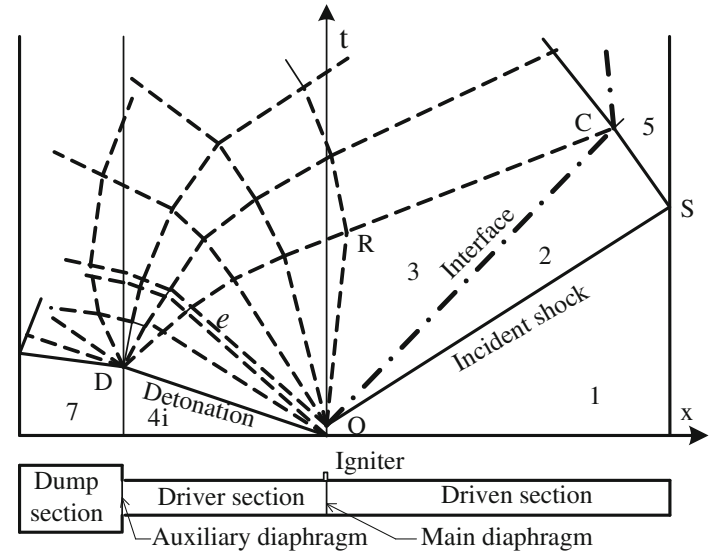

Fig. 4 Schematic diagrams of the detonation-driven chemical shock tube and wave diagram

as Glick proposed. But the two diaphragms are ruptured in rapid succession by the detonation wave itself, and the time interval between the events depends on the detonation speed and the driven section length. Since the detonation speed can be predicted correctly and shows a good repeatability [12], it can be anticipated that the detonation-driven chemical shock tube not only preserves the advantage of the shock tube proposed by Glick, but also overcomes the difficulty of the timing for the rupture of the two diaphragms.

\subsection{Expansion waves for cooling the test gas}

In a chemical shock tube, both the expansion wave from the end of the driver section and that from the shock-interface interaction can be used to cool the test gas. The former is usually used for the shock tube proposed by Glick [13], which means the shock tube needs to be operated at tailored conditions that are achieved when

$\frac{a_{3}}{\sqrt{\gamma_{3}\left[\gamma_{3}-1+\left(\gamma_{3}+1\right) P_{52}\right]}}=\frac{a_{2}}{\sqrt{\gamma_{2}\left[\gamma_{2}-1+\left(\gamma_{2}+1\right) P_{52}\right]}}$

and

$P_{52}=\frac{\left(3 \gamma_{1}-1\right) M_{\mathrm{S}}^{2}-2\left(\gamma_{1}-1\right)}{\left(\gamma_{1}-1\right) M_{\mathrm{S}}^{2}+2}$

where $a$ is the sound speed and $\gamma$ is the specific heat ratio. Subscript 2 denotes the driven gas behind the incident shock, subscript 3 the cooled driver gas and subscript 1 the test gas, and $M_{\mathrm{S}}$ is the Mach number of the incident shock. The tailored condition is suitable for providing long testing times, due to the absence of the disturbance from the interface.

The latter is usually used for the shock tube proposed by Lifshitz [13], which means that the shock tube needs to be operated at under-tailored conditions, that is 
$\frac{a_{3}}{\sqrt{\gamma_{3}\left[\gamma_{3}-1+\left(\gamma_{3}+1\right) P_{52}\right]}}>\frac{a_{2}}{\sqrt{\gamma_{2}\left[\gamma_{2}-1+\left(\gamma_{2}+1\right) P_{52}\right]}}$

The greater the difference between the value of the left side and right side in the above equation, the greater is the deviation from the tailored condition, and a stronger expansion wave is generated when the reflected shock meets the gas interface. The under-tailored condition is suitable for producing high cooling rates for the test gas, because of the shorter travel distance of the expansion wave. But the decrease of the test gas temperature is restricted by the strength of the expansion wave.

The criteria above also define the highest achievable temperature of the test gas for a certain chemical shock tube. For example, for $\mathrm{H}_{2}$ driving air at room temperature, the incident shock Mach number for tailored condition is about 6.02. This means the highest temperature of air without suffering reheating caused by the shock wave from the shock-interface interaction is less than $4000 \mathrm{~K}$. To achieve a higher-temperature condition, a higher sound speed of the driver gas is necessary.

For the proposed detonation-driven chemical shock tube, the principle of cooling the test gas is the same as that for the shock tube proposed by Glick and Lifshitz, namely, utilizing the expansion wave from the end of the driver section and that from the interface to cool the test gas. But a wider temperature range of the test gas can be obtained, because the sound speed of the driver gas for a detonation driver can be much higher than that of a conventional high-pressure driver [8, 10]. According to its applications in high-enthalpy shock tunnels $[10,12]$, for the detonation-driven chemical shock tube, it is possible to reach temperatures of the test gas up to $6000 \mathrm{~K}$ for pressures of a few to several hundred atmospheres.

\section{Operating condition}

\subsection{Optimum condition}

As mentioned above, when the shock tube operates at undertailored condition, an expansion wave is generated by the shock-interface interaction. If the test gas is cooled only by this expansion wave, for some conditions the decrease of temperature may not be enough, especially at near-tailored conditions. To achieve a sufficiently low temperature, it is necessary to cool the test gas by the expansion waves from the interface and from the auxiliary diaphragm jointly. To match this condition, the interface (line OC), the reflected shock (line SC) and the head of the expansion wave (curve DRC) starting from the auxiliary diaphragm station need to intersect at the same location, as shown in Fig. 4. In the present article, the above condition is defined as the optimum

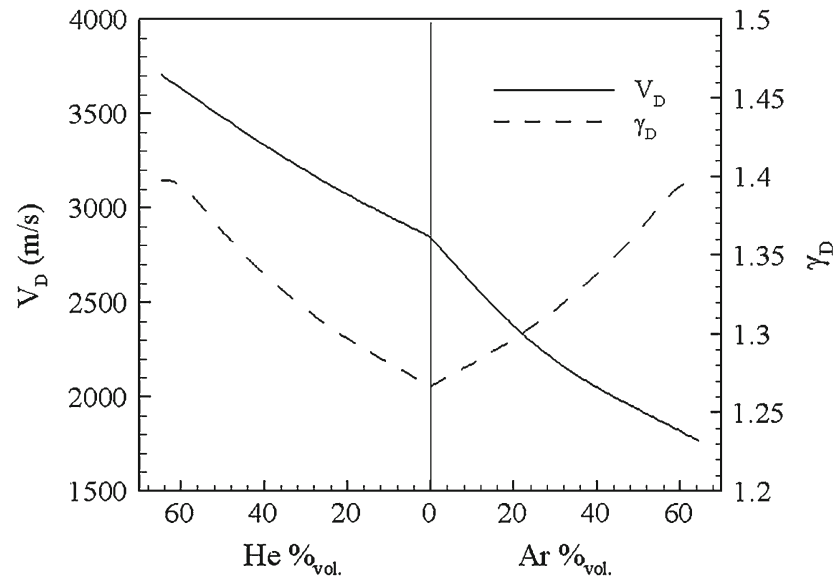

Fig. 5 Detonation speed and specific heat ratio versus concentration of helium or argon in a stoichiometric oxyhydrogen mixture, initial conditions $T_{1}=295 \mathrm{~K}, P_{1}=0.1 \mathrm{MPa}$

condition. Based on the $\mathrm{C}-\mathrm{J}$ theory and the shock tube theory, the relation of the operating parameters of the detonation-driven chemical shock tube for the optimum condition can be expressed as follows (see Appendix B)

$$
\begin{aligned}
\frac{L_{4}}{L_{1}}= & \frac{-V_{\mathrm{D}}}{2 a_{1} M_{\mathrm{S}}} \frac{\left(3 \gamma_{1}-1\right) M_{\mathrm{S}}^{2}+3-\gamma_{1}}{2 \gamma_{1} M_{\mathrm{S}}^{2}-\gamma_{1}+1} \\
& \times\left[\frac{\gamma_{\mathrm{D}}+1}{2 \gamma_{\mathrm{D}}}\left(1-\frac{2 a_{1}\left(\gamma_{\mathrm{D}}-1\right)}{-V_{\mathrm{D}}\left(\gamma_{1}+1\right)} \frac{M_{\mathrm{S}}^{2}-1}{M_{\mathrm{S}}}\right)\right]^{\frac{\gamma_{\mathrm{D}}+1}{2\left(\gamma_{\mathrm{D}}-1\right)}}
\end{aligned}
$$

where $L_{4}$ and $L_{1}$ are the lengths of the driver and driven section, respectively, $a_{1}$ is the sound speed of the driven gas and $V_{\mathrm{D}}$ is the velocity of the detonation wave in the driver section $\left(V_{D}<0\right)$, respectively. $\gamma_{1}$ and $\gamma_{D}$ are the ratios of specific heat of the test gas and the detonated driver gas, and $M_{\mathrm{S}}$ is the Mach number of the incident shock, which can be determined by [14]

$$
\begin{aligned}
\frac{P_{\mathrm{e}}}{P_{1}}= & \left(\frac{2 \gamma_{1}}{\gamma_{1}+1} M_{\mathrm{S}}^{2}-\frac{\gamma_{1}-1}{\gamma_{1}+1}\right) \\
& \times\left[1-\frac{\gamma_{\mathrm{D}}-1}{\gamma_{1}+1} \frac{a_{1}}{a_{\mathrm{e}}}\left(M_{\mathrm{S}}-\frac{1}{M_{\mathrm{S}}}\right)\right]^{\frac{2 \gamma_{\mathrm{D}}}{\gamma_{\mathrm{D}}-1}}
\end{aligned}
$$

where $P_{\mathrm{e}}$ and $a_{\mathrm{e}}$ are the effective pressure and effective sound speed of the driver gas, and $P_{1}$ is the initial pressure of the test gas.

The strength of the incident shock is usually determined by experimental demands and often needs to be changed in a certain range. Equation (4) indicates that there are two ways for changing the strength of the incident shock for the optimum condition-modifying the condition of the test gas $P_{1}$ and $a_{1}$ or adjusting the detonation speed, which together with the initial pressure $P_{4}$ determines the effective pressure $P_{e}$. The latter can be fulfilled by adjusting the initial composition of the driver gas, based on the fact that the detonation speed 


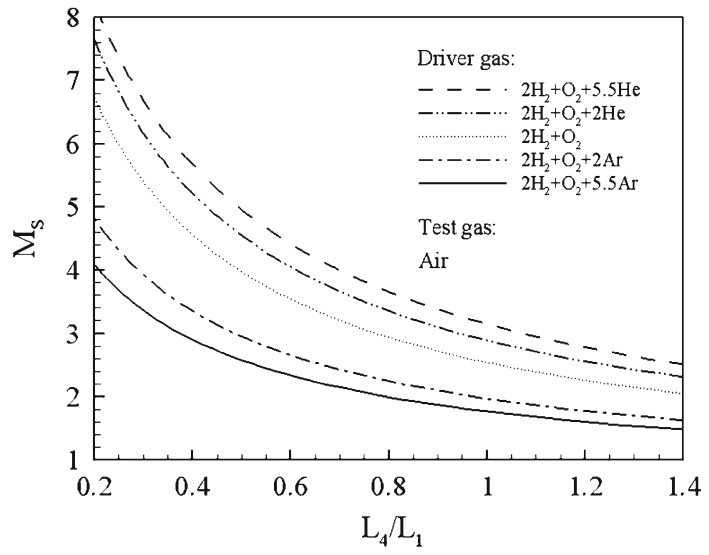

Fig. 6 Incident shock Mach numbers versus length ratios $L_{4} / L_{1}$ for different driver gases and optimum condition

and the chemical state of the gas after detonation depend strongly on the initial composition but slightly on the initial pressure [12].

For a mixture of $\mathrm{H}_{2}$ and $\mathrm{O}_{2}$, which is often used as the initial driver gas in the detonation driver, helium is usually added to achieve a high detonation speed, whereas argon dilution yields a low one. According to practical detonation initiation limits, the dilution with helium or argon to the stoichiometric oxyhydrogen mixture is limited to $65 \%$ by volume [12]. Within this limit, the detonation speeds and the ratio of specific heat of the gas after detonation for the stoichiometric oxyhydrogen mixtures diluted by the helium or argon are calculated by using the chemical equilibrium program STANJAN [15] and presented in Fig. 5. From this figure and Eq. (3), it is easy to determine the relation between the incident shock Mach number and the length ratio $L_{4} / L_{1}$ for the optimum condition for a given test gas, as shown in Fig. 6, where the test gas is air at $295 \mathrm{~K}$ with a constant specific heat ratio $\gamma_{1}=1.4$. Figure 6 indicates that for the optimum condition, the incident shock Mach number decreases as the length ratio $L_{4} / L_{1}$ or the argon fraction increases, and increases as the helium fraction increases.

Since the lengths of driver and driven section for a shock tube are usually fixed in practice, it is obvious that adjusting the initial composition of the driver gas to change the strength of the incident shock is more preferable and practicable. However, in this case the range of the strength of the incident shock is limited. For example, as shown in Fig. 6, if the length ratio is $L_{4} / L_{1}=0.6$, for the optimum condition the maximum incident shock Mach number is 4.41 for a mixture of $2 \mathrm{H}_{2}+\mathrm{O}_{2}+5.5 \mathrm{He}$ as driver gas, and the minimum value is 2.33 for a mixture of $2 \mathrm{H}_{2}+\mathrm{O}_{2}+5.5 \mathrm{Ar}$.

\subsection{Tailored condition}

The detonation-driven chemical shock tube can also be operated in the tailored condition, which is widely used in shock

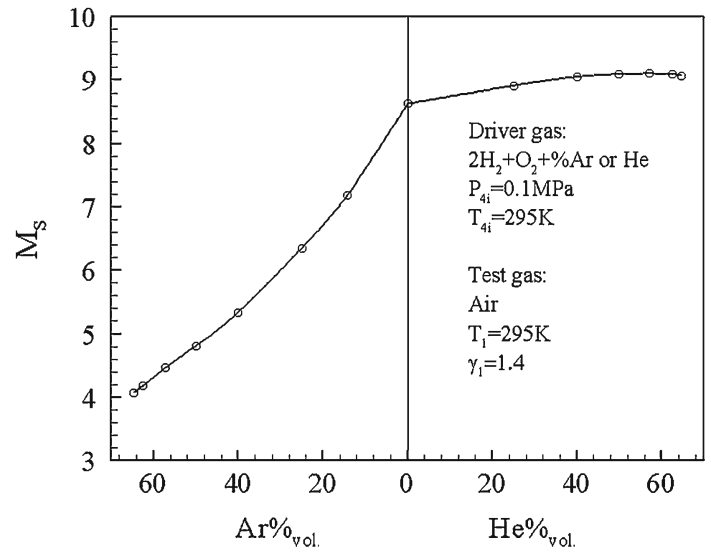

Fig. 7 Incident shock Mach number versus concentration of helium or argon dilution for stoichiometric oxyhydrogen for tailored conditions in a detonation-driven shock tube

tunnels to achieve a long testing time. In this case, the test gas is solely cooled by the expansion wave resulting from the auxiliary diaphragm. For the detonation-driven shock tube, the variations of the incident shock Mach number with the concentration of the helium or argon dilution for stoichiometric oxyhydrogen for the tailored condition are calculated based on Eq. (2) [16], as shown in Fig. 7. As for the conventional shock tube, for achieving the tailored condition, the strength of the incident shock can be adjusted by the initial composition of the driver gas. But it is particularly worth noting that there exists a lower limit for the incident shock Mach number for the tailored condition, in contrast to the absence of this limit for conventional shock tubes. This means the proposed chemical shock tube cannot provide long testing times for the test gas at low temperatures. This means that this shock tube configuration is not suitable for chemical kinetic studies that require low temperatures and long testing times simultaneously, such as ignition delay time studies at low temperatures.

In practice, it is inevitable that for certain cases the shock tube operates in off-design conditions, which deviate from the optimum condition. The consequences of this will be discussed in the following.

\section{Numerical simulations}

To validate the concept of the chemical shock tube driven by a detonation and to better understand its wave process, numerical simulations were conducted utilizing the code developed by Li et al. [17]. This numerical method is based on a onedimensional chemical nonequilibrium flow model, in which a dispersion controlled dissipation scheme [18] is adopted to solve the Euler equations, and the two-step chemical reaction model modified by Sichel et al. [19] is adapted to describe 
Table 1 Initial test gas pressures, and ideal and numerically computed shock Mach numbers

\begin{tabular}{llll}
\hline & $P_{1}(\mathrm{kPa})$ & $M_{\mathrm{i}}$ & $M_{\mathrm{c}}$ \\
\hline Case 1 & 8.3 & 4.71 & 4.66 \\
Case 2 & 0.68 & 8.91 & 8.61 \\
Case 3 & 3.70 & 5.94 & 5.79 \\
Case 4 & 12.5 & 4.16 & 4.13 \\
\hline
\end{tabular}

the detonation process in the driver gas. For simplicity, in the present paper the detonated driver gas and the test gas are assumed to be chemically frozen, which will not be of significant influence on the wave processes in most of the cases [17].

To examine the possible wave processes in the chemical shock tube driven by a detonation, four cases have been simulated with the code mentioned above. Case 1 corresponds to a optimum condition, and case 2 to the tailored condition. Cases 3 and 4 represent two conditions deviating from the optimum condition.

In all cases, the shock tube is considered as a constant cross section configuration, where the driver and driven sections are 0.4 and $1.0 \mathrm{~m}$ in length, respectively. The damping section is assumed to be long enough that the reflected waves from its end wall are neglected. Air is considered as test gas and a mixture of $2 \mathrm{H}_{2}+\mathrm{O}_{2}$ as driver gas. The initial temperature for all gases is $295 \mathrm{~K}$ and the initial driver gas pressure is fixed at $P_{4 i}=0.1 \mathrm{MPa}$. The desirable conditions are obtained by the change of the initial test gas pressure $P_{1}$ according to Eqs. (1), (3) and (4), with $V_{\mathrm{D}}=-2842 \mathrm{~m} / \mathrm{s}, P_{\mathrm{CJ}}=1.9 \mathrm{MPa}, \gamma_{\mathrm{D}}=$ 1.22 and $\gamma_{1}=1.4$. The initial pressures of the test gas, as well as the ideal and numerically computed incident shock Mach numbers $\left(M_{\mathrm{i}}\right.$ and $\left.M_{\mathrm{c}}\right)$ for the four cases are listed in Table 1.

For all cases, the ideal incident shock Mach number is slightly greater than the numerical value, and the disagreement between them tends to increase as the incident shock Mach number increases. This is mainly because the gases are in thermodynamic equilibrium (specific heat ratio depends on temperature) for the numerical simulations in contrast to the ideal flow.

The wave processes in the chemical shock tube are obvious by the computed $x-t$ diagrams representing iso-density lines, as shown in Fig. 8. From these figures, it is found that in all cases the wave processes are similar before the interactions of the reflected shock, interface and the expansion wave from the auxiliary diaphragm. The essential differences in the four cases mainly lie in the relative locations at which the waves intersect.

In case 1, after processing the test gas, the reflected shock interacts with the interface and the head of the expansion wave from the auxiliary diaphragm at almost the same location (Fig. 8a). This shock-interface interaction generates two waves. One is a transmitted shock wave, which propagates through the driver gas and the other is an expansion wave, which merges with that from the auxiliary diaphragm and propagates through the test gas. In this case, the test gas is cooled by this merged expansion wave. The pressure history at the end of the driven section is presented in Fig. 9a. At first, the arrival of the incident shock results in a pressure jump, then the pressure remains at a constant value until the arrival of the head of the merged expansion wave, and finally the pressure decreases monotonously to a low level. The waveform obtained in this case confirms that the test gas is heated by a desirable single-pulse wave if the shock tube operates in the optimum condition. The achieved testing time of 0.2 $\mathrm{ms}$, of course, also results from the length of the driver and driven section, which in this case amounts to 0.4 and $1.0 \mathrm{~m}$, respectively. The useful testing time increases with increasing length of the shock tube parts.

In case 2 , because the shock tube operates in the tailored condition, the reflected shock passes through the interface leaving behind only a Mach wave, which does not perturb the uniform state of the test gas, and the interface is abruptly brought to rest by this interaction (Fig. 8b). The uniform state of the test gas persists until the arrival of the head of the expansion wave from the auxiliary diaphragm, so that a longer testing time is observed. But in this case, the rate of pressure decrease and therewith the cooling rate is lower in contrast to the last case, as shown in Fig. 9b. This result is consistent with the discussion in Sect. 4.

In case 3, the reflected shock meets the interface prior to the arrival of the head of the expansion wave from the auxiliary diaphragm (Fig. 8c), the consequence of which is that the test gas is cooled by the expansion waves from the interface and the auxiliary diaphragm separately with a high and a low cooling rate. As Fig. 9c shows, at approximately $0.8 \mathrm{~ms}$, a weak pressure increase with subsequent decrease occurs caused by the interaction of the interface with the reflected shock. This is in contrast with the smooth decrease for the first two cases. According to the discussion in Sect. 4.1, to relieve or avoid this situation, it is required to shorten the driver section or increase the detonation speed if the strength of the incident shock is fixed.

In case 4 , the interface is overtaken by the head of the rarefaction from the auxiliary diaphragm before intersecting with the reflected shock wave (Fig. 8d). As a result, part of the test gas is cooled by the expansion wave before it can be heated by the reflected shock wave. At the end of the useful testing time, the initial rate of the pressure decrease is less compared to cases 1 and 3, as shown in Fig. 9d, but the feature of a single pulse remains. This behavior can be relieved or avoided by lengthening the driver section or decreasing the detonation speed.

The wave processes examined in the four cases represent the majority of cases that may occur for a detonation-driven 


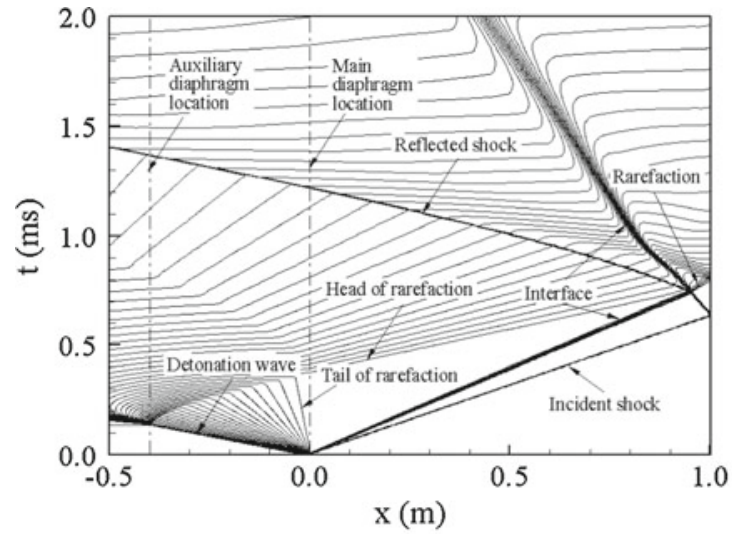

(a) Case 1

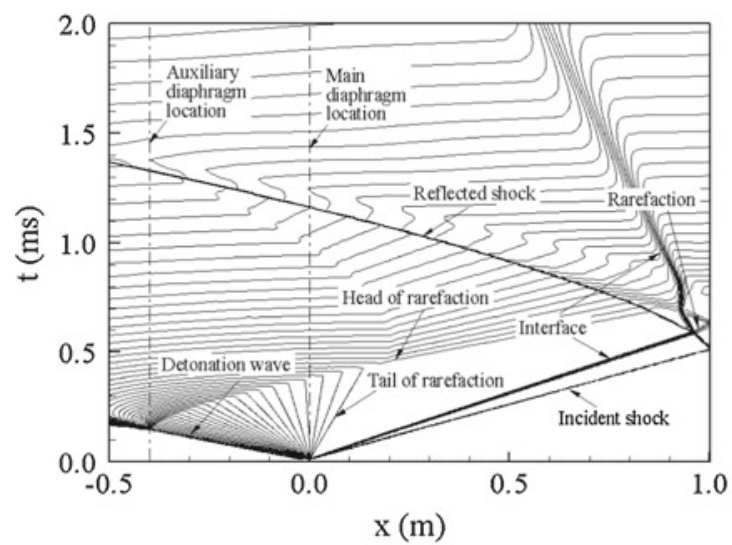

(c) Case 3

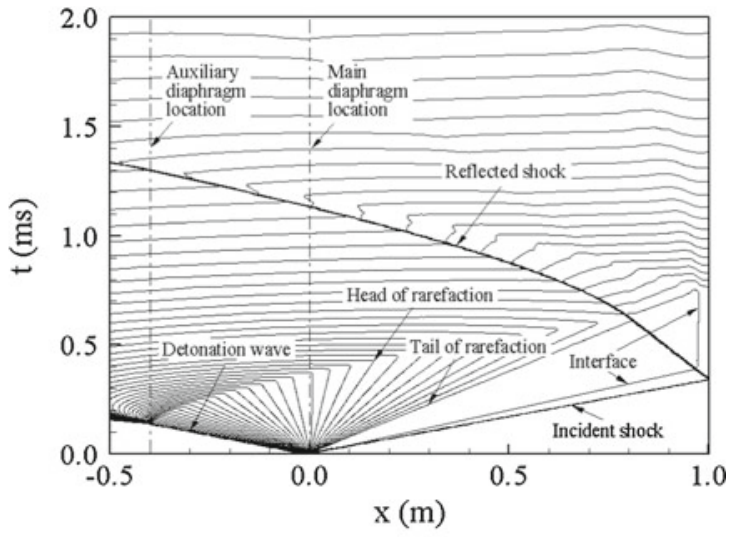

(b) Case 2

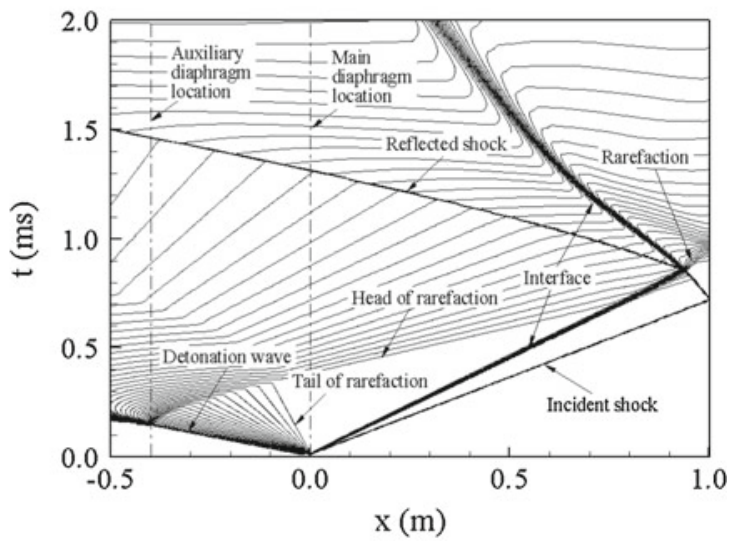

(d) Case 4

Fig. 8 Computed $x-t$ diagrams of density contours of the chemical shock tube driven by a detonation wave

Fig. 9 Computed pressure histories at the end of the driven section for four cases

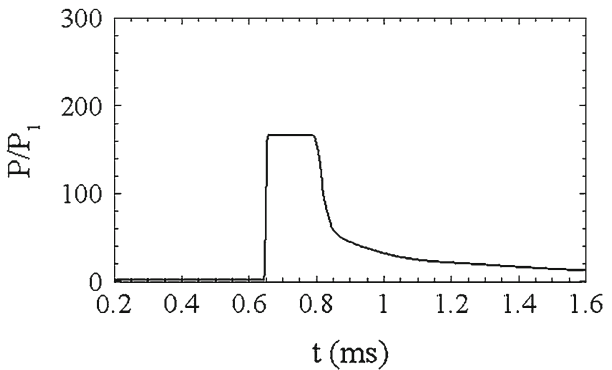

(a) Case 1

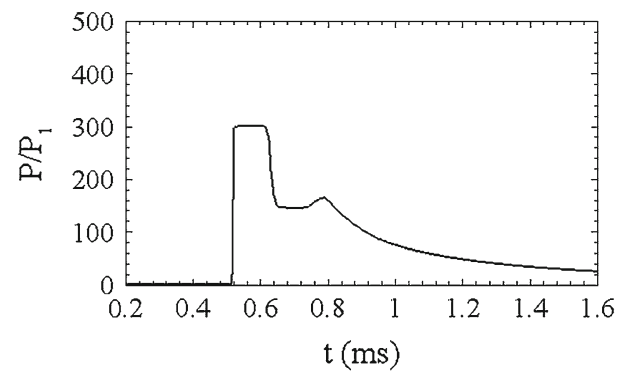

(c) Case 3

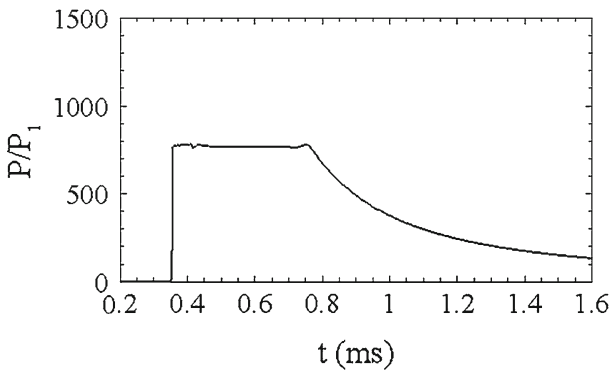

(b) Case 2

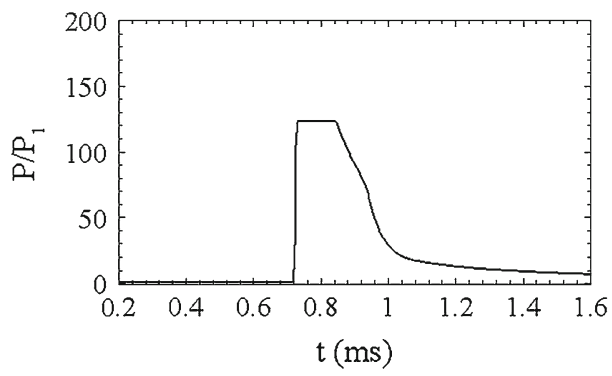

(d) Case 4 
Fig. 10 Schematic diagram of the detonation-driven chemical shock tube in LHD

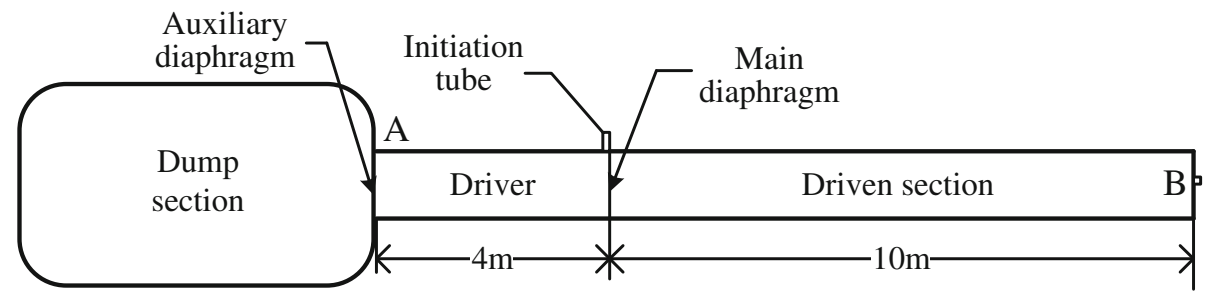

Table 2 Operating conditions

\begin{tabular}{llllllll}
\hline & \multicolumn{2}{l}{ Driver section } & & & & Driven section & \\
\cline { 2 - 3 } & Composition & Pressure $(\mathrm{MPa})$ & Temperature $(\mathrm{K})$ & & Composition & Pressure $(\mathrm{kPa})$ & Temperature $(\mathrm{K})$ \\
\hline Condition 1 & $2 \mathrm{H}_{2}+\mathrm{O}_{2}$ & 0.08 & 292 & Air & 7.0 & 292 \\
Condition 2 & $2 \mathrm{H}_{2}+\mathrm{O}_{2}+\mathrm{N}_{2}$ & 0.08 & 291 & Air & 9.9 & 291 \\
\hline
\end{tabular}

Fig. 11 Experimental pressure histories for two runs for condition 1

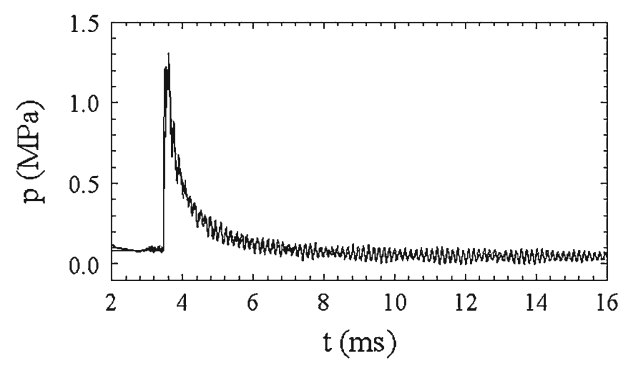

(a) Position $\mathrm{A}$

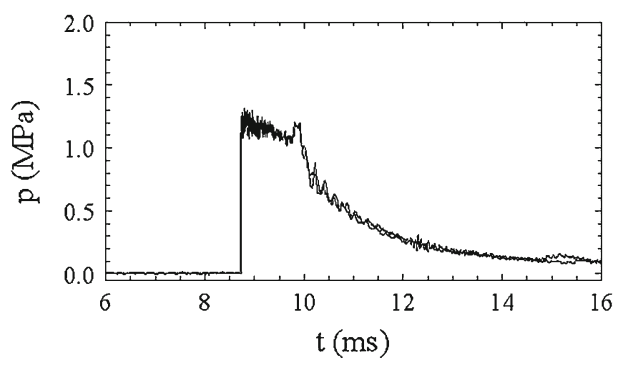

(b) Position B chemical shock tube. As far as the wave process is concerned, the influence of the detonation speed of the driver gas and the length ratio $L_{4} / L_{1}$ is similar to that of the strength of the incident shock, which will not be discussed in detail.

The results achieved by the numerical simulation show that the detonation-driven chemical shock tube works well for the optimum and tailored condition. For off-design conditions, the performance of the shock tube degrades. But, conditions slightly deviating from the optimum condition should be acceptable, because the waveforms obtained for these conditions still remain the features of a single pulse.

\section{Experimental results}

In order to examine the feasibility of a chemical shock tube driven by a detonation wave in practice, experiments were carried out in the Laboratory of High Temperature Gas Dynamics (LHD), Institute of Mechanics, Beijing, China. The shock tube consists of a dump section, a driver section and a driven section, as shown in Fig. 10. The driver section is $4.05 \mathrm{~m}$ long and $224 \mathrm{~mm}$ in diameter. The driven section is $10.2 \mathrm{~m}$ long and $224 \mathrm{~mm}$ in diameter, and the dump section is $17 \mathrm{~m}$ long and $500 \mathrm{~mm}$ in diameter. Both the main and the auxiliary diaphragm are $0.2 \mathrm{~mm}$ thick and made from Terylene, which is sufficient because the initial pressure differences across the diaphragms are not large. An initiation tube is used for igniting the detonable gas. It is placed perpendicularly to the driver tube, immediately upstream of the main diaphragm, which ensures that the detonation wave is initiated directly [20]. Two pressure transducers are mounted on the shock tube: transducer $\mathrm{A}$ is located at the driver section close to the auxiliary diaphragm, and transducer $\mathrm{B}$ is located at the end wall of the driven section.

Experiments were conducted for two different operating conditions listed in Table 2, which are expected to satisfy the optimum conditions defined in Sect. 4.1. The dump section was evacuated to a pressure of approximately $50 \mathrm{~Pa}$ before each run.

The experimental results for two runs of each condition are presented in Figs. 11 and 13, which show that the repeatability is very good. The experimental Mach number of the incident shock near the end wall of the driven section is 4.40 and 3.88 for conditions 1 and 2, respectively, which is a little lower than the corresponding ideal value of 4.71 and 3.98. This is mainly because of dissipative effects due to wall friction and heat conduction. The measured detonation speed for the two conditions is 2870 and $2450 \mathrm{~m} / \mathrm{s}$, deviating $3 \%$ from the corresponding theoretical $\mathrm{C}-\mathrm{J}$ values. 
Fig. 12 Computed pressure histories for condition 1

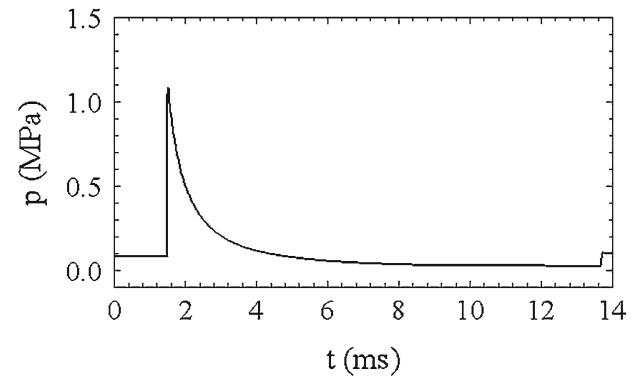

(a) Position A

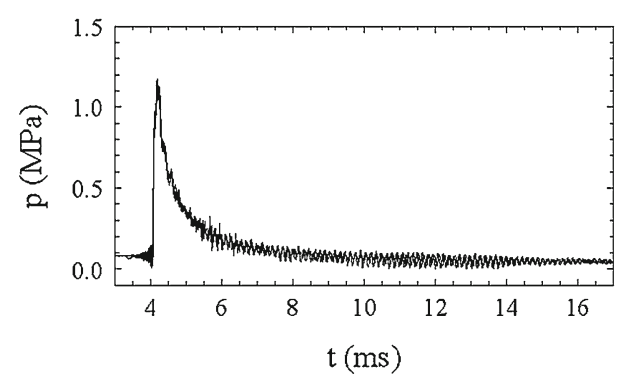

(a) Position $\mathrm{A}$

Fig. 13 Experimental pressure histories for two runs for condition 2
Fig. 14 Computed pressure histories for condition 2

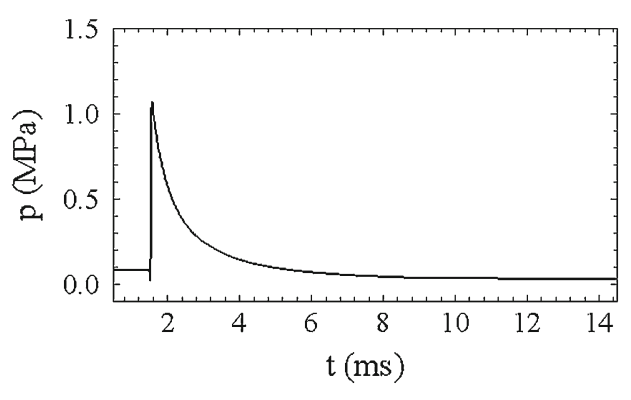

(a) Position A

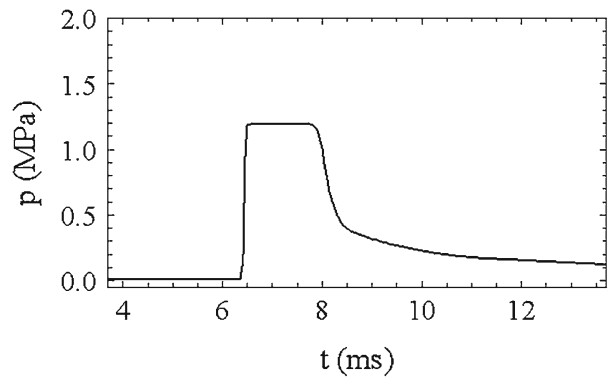

(b) Position B

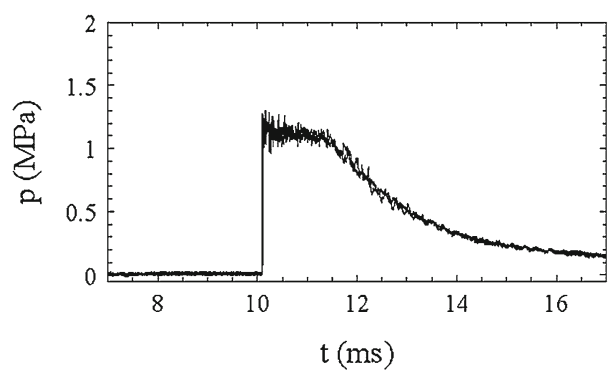

(b) Position B

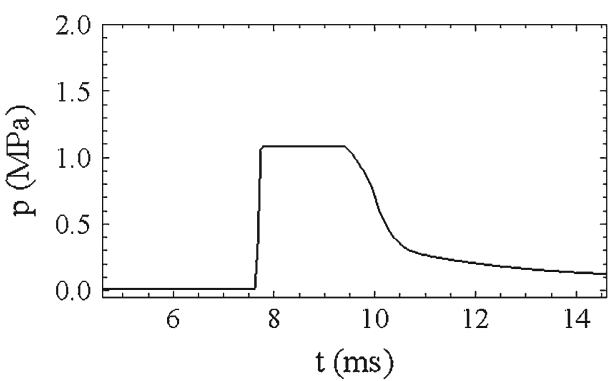

(b) Position B
Figures 11a and 13a show the pressure histories measured at position $\mathrm{A}$ for the two conditions, which agree well with the corresponding numerical results (Figs. 12a, 14a). The jump of the pressure is due to the arrival of the detonation wave and the decrease in results from the combined effect of the Taylor expansion wave and the expansion wave generated by the rupture of the auxiliary diaphragm.

The pressure variations measured at position B for the two conditions are presented in Figs. 11b and 13b, which confirm that the detonation-driven chemical shock tube can produce the desired single-pulse wave for chemical experimental studies. The pulse duration for the two conditions is approximately $1.5 \mathrm{~ms}$. However, compared to the computed results (Figs. 12b, 14b), it is found that the pulse durations are a little shorter than in the computations. This could be because the conditions in which the shock tube was actually operated slightly deviated from the optimum condition due to the attenuation of the incident shock. Therefore, the interface is overtaken by the head of the rarefaction wave from the auxiliary diaphragm before interacting with the reflected shock wave, as discussed for case 4 in Sect. 5. In addition, the rate of the pressures decrease in the experiments a little less than that in the computations, which is attributed to the diffuse character of the interface that weakens the expansion wave.

The initial cooling rate of the test gas is one of the most important parameters for the chemical shock tube, because it is the critical quantity that determines the smallest activation energy for which a reaction can be quenched [21]. Figure 15 shows the computed temperature histories at the end wall of the driven section, from which the cooling rates of the test gas are determined. The initial cooling rate is about $0.41 \times 10^{6} \mathrm{~K} / \mathrm{s}$ at $t=8.0 \mathrm{~ms}$ for condition 1 , and $1.1 \times 10^{6} \mathrm{~K} / \mathrm{s}$ at $t=9.6 \mathrm{~ms}$ for condition 2 , which is equivalent to the value of $0.5-1.0 \times 10^{6} \mathrm{~K} / \mathrm{s}$ available from the chemical shock tube proposed by Lifshitz [13]. 
Fig. 15 Computed temperature histories at the end wall of the driven section

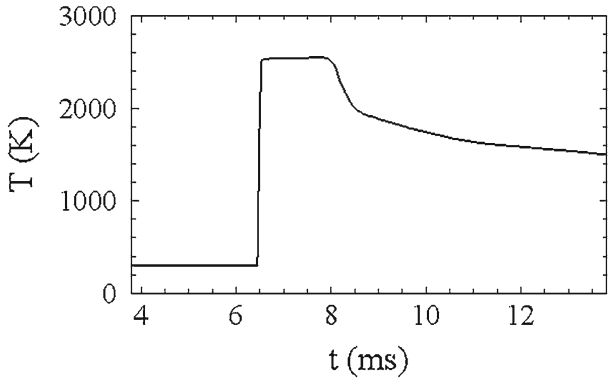

(a) Condition 1

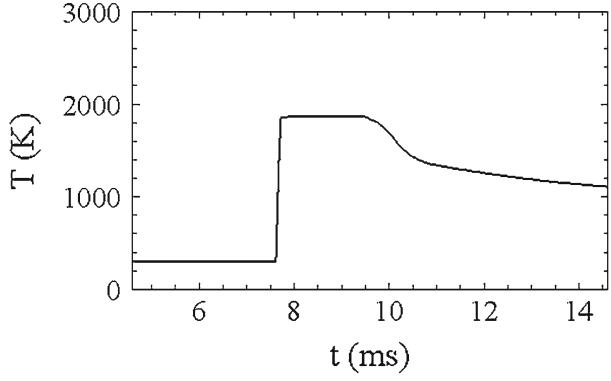

(b) Condition 2
These experimental results also demonstrate that the adjustment of the composition of the initial driver gas to vary the strength of the incident shock to achieve the optimum condition is a practicable method for the detonation-driven chemical shock tube.

\section{Conclusion}

A chemical shock tube driven by a detonation driver is proposed in the present paper. This shock tube conforms to the principle of producing single high-temperature pulses, as proposed by Glick, but the difficulty associated with the timing of the rupture of the two diaphragms is avoided. Moreover, this shock tube is capable of providing higher temperature conditions for chemical studies than the conventional high-pressure shock tube without loss of the feature of a single pulse.

It is preferable that the shock tube operates in the optimum condition or tailored condition to achieve the desirable conditions for chemical studies. The former is suitable for producing high cooling rates for the test gas, whereas the latter is suitable for long testing times. These conditions can be achieved by modifying the length ratio of driver to driven section or adjusting the initial composition of the driver gas.

There are also some limitations for this shock tube for chemical studies compared to existing chemical shock tubes. It is difficult for this shock tube to provide long testing times for the test gas at low temperatures, because it cannot be operated in tailored conditions for weak incident shocks. In addition, in some cases the driver gas may have influence on the reaction products, because it consists of the burnt products after detonation instead of usual noble gases as in conventional or other chemical shock tubes. The aim of the paper is to present and to discuss this new concept of utilizing a detonation-driven shock tube for chemical studies from the point of view of the shock tube process. Therefore, until now there are no chemical kinetic experiments being conducted with this chemical shock tube. From the proven capabilities of this method, it is expected that the technique will be applied in the near future for chemical kinetic studies. Based on these results, its flexibility and capability then need to be evaluated further.

\section{Appendix A: Effective conditions of the detonation driver}

Based on the Chapman-Jouguet detonation theory [11], if a detonation wave travels from the right to the left in a tube (Fig. 3), and the initial pressure of the detonable gas is neglected, the $\mathrm{C}-\mathrm{J}$ sate is given by

$$
\begin{aligned}
& P_{\mathrm{CJ}}=\frac{\rho_{0} V_{\mathrm{D}}^{2}}{\left(\gamma_{\mathrm{D}}+1\right)} \\
& u_{\mathrm{CJ}}=\frac{V_{\mathrm{D}}}{\gamma_{\mathrm{D}}+1} \\
& a_{\mathrm{CJ}}=-\frac{\gamma_{\mathrm{D}} V_{\mathrm{D}}}{\gamma_{\mathrm{D}}+1}
\end{aligned}
$$

where $P_{\mathrm{CJ}}, u_{\mathrm{CJ}}$ and $a_{\mathrm{CJ}}$ are the $\mathrm{C}-\mathrm{J}$ pressure, flow velocity and sound speed, $V_{\mathrm{D}}$ is the detonation velocity with $V_{D}<0$ in this case, $\rho_{0}$ is the initial density of the detonable gas, and $\gamma_{\mathrm{D}}$ is the ratio of specific heat of the gas after detonation. The accuracy of the above equations is sufficient for the estimation of the conditions in the detonation driver section.

The effective driver gas of the detonation driver is the gas decelerated to velocity zero by the Taylor wave (simple wave) from the CJ state. Hence,

$u_{\mathrm{e}}+\frac{2}{\gamma_{\mathrm{D}}-1} a_{\mathrm{e}}=u_{\mathrm{CJ}}+\frac{2}{\gamma_{\mathrm{D}}-1} a_{\mathrm{CJ}}$

where $u_{\mathrm{e}}$ and $a_{\mathrm{e}}$ are the flow velocity and sound speed on the characteristic e in Fig 4. From the above equations and using $u_{\mathrm{e}}=0$, the effective sound speed of driver gas $a_{\mathrm{e}}$ can be expressed by

$a_{\mathrm{e}}=-\frac{V_{\mathrm{D}}}{2}$ 
From the isentropic relation, the effective pressure of driver gas $P_{\mathrm{e}}$ is given by

$P_{\mathrm{e}}=P_{\mathrm{CJ}}\left(\frac{a_{\mathrm{e}}}{a_{\mathrm{CJ}}}\right)^{\frac{2 \gamma_{\mathrm{D}}}{\gamma_{\mathrm{D}}-1}}$

\section{Appendix B: Optimum condition for the chemical shock tube driven by detonation}

The detonation wave (line OD in Fig. 4) travels at a constant velocity $V_{\mathrm{D}}$ towards the upstream end of the driver section, the equation of which in the $(x, t)$ plane is thus

$x=V_{\mathrm{D}} t\left(x \geq-L_{4}\right)$

where $L_{4}$ is the length of the driver section.

The head of the expansion wave originating at the auxiliary diaphragm station (curve DR) travels at the local speed of sound relative to the flow in the region between line OD and line OR. This is a simple wave region; the equation of the head of the wave is

$\frac{\mathrm{d} x}{\mathrm{~d} t}=u+a$

and

$$
\left\{\begin{array}{l}
u-a=\frac{x}{t}\left(\frac{x}{t} \geq V_{\mathrm{D}}\right) \\
u+\frac{2}{\gamma_{\mathrm{D}}-1} a=u_{\mathrm{CJ}}+\frac{2}{\gamma_{\mathrm{D}}-1} a_{\mathrm{CJ}}=\frac{-V_{\mathrm{D}}}{\gamma_{\mathrm{D}}-1}
\end{array}\right.
$$

where $u$ is the flow velocity and $a$ is the local sound speed in the region between line OD and line OR. Based on Eq. (a.9), Eq. (a.8) can be written as

$\frac{\mathrm{d} x}{\mathrm{~d} t}=u+a=\frac{3-\gamma_{\mathrm{D}}}{\gamma_{\mathrm{D}}+1} \frac{x}{t}+\frac{-2 V_{\mathrm{D}}}{\gamma_{\mathrm{D}}+1}$

Integrating the above equation results in

$x=\frac{-V_{\mathrm{D}}}{\gamma_{\mathrm{D}}-1} t-c t^{\frac{3-\gamma_{\mathrm{D}}}{1+\gamma_{\mathrm{D}}}}$

where $c$ is an integration constant. The condition to determine this constant is $x=-L_{4}, t=-L_{4} / V_{\mathrm{D}}$. Hence, the equation of curve DR can be expressed as

$x=\frac{-V_{\mathrm{D}}}{\gamma_{\mathrm{D}}-1} t-\frac{\gamma_{\mathrm{D}}}{\gamma_{\mathrm{D}}-1} L_{4}\left(\frac{-V_{\mathrm{D}} t}{L_{4}}\right)^{\frac{3-\gamma_{\mathrm{D}}}{\gamma_{\mathrm{D}}+1}}$

The tail of the expansion wave produced by the rupture of the main diaphragm (line OR) moves with a constant velocity in the opposite direction to the incident shock. The equation of the tail of the expansion wave is

$x=\left(u_{3}-a_{3}\right) t$

where $u_{3}$ and $a_{3}$ are the flow velocity and local sound speed in region 3 (between line OR and line OC). With the relation for a right running characteristic in the simple wave region

$\frac{2}{\gamma_{\mathrm{D}}-1} a_{\mathrm{e}}=\frac{2}{\gamma_{\mathrm{D}}-1} a_{3}+u_{3}$

and Eq. (a.5), Eq. (a.13) can be written as

$x=\frac{-V_{\mathrm{D}}}{2} \frac{\left(M_{3}-1\right)}{\left(1+\frac{\gamma_{\mathrm{D}}-1}{2} M_{3}\right)} t$

where $M_{3}=u_{3} / a_{3}$, which is given by [14]

$$
\begin{aligned}
M_{3} & =\left[\frac{a_{\mathrm{e}}}{a_{1}} \frac{\left(\gamma_{1}+1\right) M_{\mathrm{S}}}{2\left(M_{\mathrm{S}}^{2}-1\right)}-\frac{\gamma_{\mathrm{D}}-1}{2}\right] \\
& =\left[\frac{-V_{\mathrm{D}}}{2 a_{1}} \frac{\left(\gamma_{1}+1\right) M_{\mathrm{S}}}{2\left(M_{\mathrm{S}}^{2}-1\right)}-\frac{\gamma_{\mathrm{D}}-1}{2}\right]
\end{aligned}
$$

where $\gamma_{1}$ is the ratio of specific heat of the test gas, $M_{\mathrm{S}}$ is the Mach number of the incident shock and $a_{1}$ is the initial speed of sound of the test gas.

The point $R\left(x_{R}, t_{R}\right)$ at which curve $\mathrm{DR}$ and line OR intersect is obtained by identifying (a.15) and (a.12), which results in

$x_{R}=\frac{L_{4}}{2}\left(M_{3}-1\right)\left(1+\frac{\gamma_{\mathrm{D}}-1}{2} M_{3}\right)^{-1}\left(\frac{1+\frac{\gamma_{\mathrm{D}}-1}{2} M_{3}}{\frac{\gamma_{\mathrm{D}}+1}{2 \gamma_{\mathrm{D}}}}\right)^{\frac{\gamma_{\mathrm{D}}+1}{2\left(\gamma_{\mathrm{D}}-1\right)}}$

$t_{R}=\frac{L_{4}}{-V_{\mathrm{D}}}\left(\frac{1+\frac{\gamma_{\mathrm{D}}-1}{2} M_{3}}{\frac{\gamma_{\mathrm{D}}+1}{2 \gamma_{\mathrm{D}}}}\right)^{\frac{\gamma_{\mathrm{D}}+1}{2\left(\gamma_{\mathrm{D}}-1\right)}}$

In region 3, the head of the expansion wave originating at the auxiliary diaphragm (line RC) moves at a constant velocity, the equation of which is

$x=x_{R}+\left(u_{3}+a_{3}\right)\left(t-t_{R}\right)$

If the driven section is long enough, the contact surface will be overtaken by the head of the expansion wave from the auxiliary at point $C\left(x_{\mathrm{c}}, t_{\mathrm{c}}\right)$. From the above equations and using $x_{R}=\left(u_{3}-a_{3}\right) t_{R}$ give

$t_{C}=\frac{2 L_{4}}{-V_{\mathrm{D}}}\left(\frac{1+\frac{\gamma_{\mathrm{D}}-1}{2} M_{3}}{\frac{\gamma_{\mathrm{D}}+1}{2 \gamma_{\mathrm{D}}}}\right)^{\frac{\gamma_{\mathrm{D}}+1}{2\left(\gamma_{\mathrm{D}}-1\right)}}$

with

$x_{C}=u_{3} t_{C}=u_{2} t_{C}$

where $u_{2}$ is the flow velocity in region 2 (between line OS and line $\mathrm{OC}$ ), which is determined by

$u_{2}=\frac{2 a_{1}\left(M_{\mathrm{S}}^{2}-1\right)}{M_{\mathrm{S}}\left(\gamma_{1}+1\right)}$ 
The equation for the reflected shock wave (line SC) in the $(x, t)$ plane is

$$
\begin{aligned}
x= & L_{1}\left(\frac{3 \gamma_{1}-1}{\gamma_{1}+1}+\frac{3-\gamma_{1}}{\gamma_{1}+1} \frac{1}{M_{\mathrm{S}}^{2}}\right) \\
& -\left[\frac{2\left(\gamma_{1}-1\right)}{\gamma_{1}+1}+\frac{3-\gamma_{1}}{\gamma_{1}+1} \frac{1}{M_{\mathrm{S}}^{2}}\right] M_{\mathrm{S}} a_{1} t
\end{aligned}
$$

The optimum condition means that the reflected shock wave also passes through point $C\left(x_{\mathrm{c}}, t_{\mathrm{c}}\right)$. Substituting Eqs. (a.20) and (a.21) into Eq. (a.23) yields

$$
\begin{aligned}
\frac{L_{4}}{L_{1}}= & \frac{-V_{\mathrm{D}}}{2 a_{1} M_{\mathrm{S}}} \frac{\left(3 \gamma_{1}-1\right) M_{\mathrm{S}}^{2}+3-\gamma_{1}}{2 \gamma_{1} M_{\mathrm{S}}^{2}-\gamma_{1}+1} \\
& \times\left[\frac{\gamma_{\mathrm{D}}+1}{2 \gamma_{\mathrm{D}}}\left(1-\frac{2 a_{1}\left(\gamma_{\mathrm{D}}-1\right)}{-V_{\mathrm{D}}\left(\gamma_{1}+1\right)} \frac{M_{\mathrm{S}}^{2}-1}{M_{\mathrm{S}}}\right)\right]^{\frac{\gamma_{\mathrm{D}}+1}{2\left(\gamma_{\mathrm{D}}-1\right)}}
\end{aligned}
$$

\section{References}

1. Greene, E.F., Taylor, R.L., Patterson, W.L. Jr.: Pyrolysis of simple hydrocarbons in shock waves. J. Phys. Chem. 62, 238-243 (1958)

2. Marshall, R., Davidson, N.: Photoelectric observation of the rate of recombination of iodine atoms. J. Chem. Phys. 21, 659-664 (1953)

3. Glick, H.S., Squire, W., Hertzberg, A.: A new shock tube technique for the study of high temperature gas phase reactions. In: The Fifth (International) Symposium on Combustion, Pittsburg, Pennsylvania, August 30-September 3, 1954, pp. 393-402. Reinhold, New York (1955)

4. Lifshitz, A., Bauer, S.H., Resler, E.L.: Studies with single-pulse shock tube. I. The cis-trans isomerization of betene-2. J. Chem. Phys. 38, 2056-2063 (1963)

5. Fang, B.C., Cui, J.P.: Single-pulse tube with a magic hole. Aerodyn. Exp. Meas. Control 4, 58-62 (1990)

6. Bird, G.A.: A note on combustion driven shock tubes. AGARD Report No. 146 (1957)
7. Lee, B.H.K.: Detonation driven in a shock tube. AIAA J. 5(4), 791-792 (1967)

8. Yu, H.R., Esser, B., Lenartz, M., Groenig, H.: Gaseous detonation driver for a shock tunnel. Shock Waves 2(4), 245-254 (1992)

9. Erdos, J.I., Bakos, R.J., Rogers, R.C.: Dual mode shock expansion/reflected-shock tunnel. AIAA Paper 97-0560 (1997)

10. Lu, F.K., Wilson, D.R., Bakos, R.J., Erdos, J.I.: Recent advances in detonation techniques for high-enthalpy facilities. AIAA J. 38(9), 1676-1684 (2000)

11. Fickett, W., Davis, W.C.: Detonation Theory and Experiment. Dover, Mineola (1979)

12. Olivier, H., Jiang, Z.L., Yu, H.R.: Detonation-driven shock tubes and tunnels. Advanced hypersonic test facilities. In: Lu, F., Marren, D. (eds.) Progress in Astronautics and Aeronautics, vol. 198, pp. 135-205. AIAA (2002)

13. Lifshitz, A.: The Single Pulse Shock: Its Odyssey in Chemical Kinetics. In: Jiang, Z. (ed.) Proceedings of the 24th International Symposium on Shock Waves, pp. 57-64, Beijing, July 11-16, 2004 (2004)

14. Gaydon, A.G., Hurle, I.R.: The Shock Tube in High-Temperature Chemical Physics. Chapman and Hall, London (1963)

15. Reynolds, W.C.: The Element Potential Method for Chemical Equilibrium Analysis: Implementation in the Interactive Program STANJAN, Version 3. Mechanical Engineering Department, Stanford University (1986)

16. Li, J.P., Feng, H., Jiang, Z.L.: Numerical computation on the tailored shock mach numbers for a hydrogen-oxygen detonation shock tube. Acta Aerodyn. Sin. 26(3), 291-296 (2008)

17. Li, J.P., Jiang, Z.L., Chen, H.: Numerical study on backwardforward double detonation driver for high-enthalpy shock tubes. Chin. J. Theor. Appl. Mech. 39(3), 343-349 (2007)

18. Jiang, Z.L., Takayama, K., Chen, Y.S.: Dispersion conditions for non-oscillatory shock capturing schemes and its applications. Comput. Fluid Dyn. J. 4(2), 137-150 (1995)

19. Sichel, M., Tonello, N.A., Oran, E.S., Jones, D.A.: A two-step kinetics model for numerical simulation of explosion and detonation in $\mathrm{H}_{2}-\mathrm{O}_{2}$ mixtures. Proc. R. Soc. Lond. A 458, 49-82 (2002)

20. Zhang, X.Y., Yu, H.R.: Turbulent Jet Initiation of Oxy-hydrogen Direct Detonation. Aerodyn. Exp. Measur. Control 10(2), 63-68 (1996)

21. Hertzberg, A., Glick, H.S.: Kinetics studies in a single-pulse shock tube. In: Ferri, A. (ed.) Fundamental Data Obtained from ShockTube Experiments, pp. 161-182. Pergamon Press, London (1961) 